\title{
Outgrowing the Procrustean Bed of Normality: The Utility of Bayesian Modeling for Asymmetrical Data Analysis
}

\author{
Stephen R. Martin \\ Baylor University
}

\author{
Donald R. Williams \\ University of California, Davis
}

\begin{abstract}
Psychological data often violate the normality assumptions made by commonly used statistical methods. These violations are addressed in a variety of ways such as transformations or assuming the employed method is robust to violations. Here we argue that data transformations are unnecessary at best and severely misleading at worst. An alternative approach is to use a Bayesian model that permits skewness and other perturbations to classical assumptions (e.g., heteroskedasticity). Through simulation, we demonstrate that a Bayesian skew-normal model has optimal frequentist properties (i.e., "type 1" error, "power", unbiasedness) compared to normal-assumptive models with or without transformation. Furthermore, the Bayesian skewnormal model has greater predictive utility, as indicated by posterior predictive checking and approximate leave-one-out cross-validation. After an applied example, we discuss practical implications of our findings for psychological science in general, and specifically how Bayesian modeling can improve reproducibility in psychology.
\end{abstract}

Keywords: Bayesian, skew, normality, power, bias, error

The single most important formula in psychological science is the normal probability density function:

$$
f\left(x_{i}, \mu, \sigma\right)=\frac{1}{\sqrt{2 \pi \sigma^{2}}} e^{-\frac{\left(x_{i}-\mu\right)^{2}}{2 \sigma^{2}}}
$$

Since the introduction of quantitative methods in psychology, descriptive statistics have been computed and sampling distributions derived under the assumption of normality. From simple z-scores up to multilevel models and latent continua, the normal distribution has served psychologists well with its useful and well defined properties. While the importance of the normal distribution cannot be understated, we take the position that researchers have become too dependent on assuming their outcome variables are normally distributed. There are hundreds of probability distributions that can feasibly model one's data, and yet normality is commonly treated as a necessary goal that lacks a theoretical motivation. While

Affiliations Stephen R. Martin, Department of Psychology and Neuroscience, Baylor University; Donald R. Williams, Department of Psychology, University of California, Davis.

Acknowledgments We extend our gratitude to Dr. Masicampo at Wake Forest University for his role in collecting and permitting use of the data used in the motivating example.

Corresponding Author Stephen R. Martin (Stephen_Martin@Baylor.edu), Dept. of Psychology and Neuroscience, Baylor University, One Bear Place, Waco, TX, 97310 in the past fitting statistical models required simplifying assumptions, modern computational advancements allow for fitting better models that are more robust and generalized than could have been fit in the 20th century. Due to these advancements, we argue for and demonstrate the utility of Bayesian modeling, in particular for asymmetrical data analysis (i.e., skewed data).

To explain our perspective, we first introduce the notion of the data generating process. We then discuss practical and theoretical issues with non-linear data transformations with respect to the data generating process. The basic tenets and flexibility of Bayesian modeling are introduced. The skewnormal distribution is described, and through simulation we demonstrate that the skew-normal model can have nominal "type 1" error rates, optimal power, negligible bias, and optimal predictive performance. An example on real data is provided, with accompanying annotated code for three packages in the appendices.

\section{Data Generating Process}

A data generating process (DGP) is defined here as the probabilistic system through which observations are realized. All inferential models are, ultimately, seeking information about the DGP. As researchers, our goal is to better understand, statistically model, and predict future events from the modeled DGP. Importantly, all statistical models are quantitative representations of the presumed DGP from which we derive our inferences about reality.

Modeling the DGP does not imply or require advanced techniques (e.g., SEM). Take one of the simplest of modeling 
techniques, the student t-test for a mean differences, as an example. Here, one assumes data from two groups are random, independent variates generated by normal distributions with a total of three parameters: $\mu_{1}, \mu_{2}, \sigma$. Of course, the presumed generating parameters of the DGP are unknown and must estimated from the data with $\overline{x_{1}}, \overline{x_{2}}, \hat{\sigma}$. The hypothesis is that the parameters underlying the DGP for the two groups are different, $\mu_{1} \neq \mu_{2}$. In a NHST framework, a "null" DGP is posited, $H_{0}: \mu_{1}=\mu_{2} ; \mu_{1}-\mu_{2}=0$, and the goal is to infer whether such a DGP could feasibly generate observed data, given the estimates of the parameters. Given an assumption of normality, independence of observations, and homogenous variance, a t statistic is computed, $\frac{\overline{x_{1}}-\overline{x_{2}}-\left(\mu_{1}-\mu_{2}\right)}{\hat{\sigma}_{\mu_{1}}-\mu_{2}}$, that has a known sampling distribution - the $t$ distribution with degrees of freedom equal to $n-2$. The probability of obtaining a value greater than the computed $t$ statistic given the null DGP may be small, leading to an inference that the DGP generates groups with differing means. This is to say, even when using a simple model, inferences are made about an assumed DGP.

\section{Model Assumptions}

One must make assumptions when modeling the DGP. However, the purposes of these assumptions are not fully appreciated and are seemingly neglected by researchers. Assumptions serve two primary purposes. First, when assumptions are met, estimation and inferential procedures are simplified. Assumptions allow for unknowns and nuisance parameters to cancel or be otherwise ignored. Simplifying models through assumptions may reduce them to a closed form that is analytically tractable. For example, when homoskedastic, independent, normally distributed data are observed the sampling distribution of the t-statistic is known, and thus the Student t-test can be used with the desired frequentist properties. Although these properties were useful in an era void of computers, they are less relevant with the modern computational power to estimate empirical sampling distributions and highly multidimensional models.

The second purpose is a theoretical one, such that assumptions are proposed components of the DGP, and thus comprise one's model. That is, assumptions encode how observations and unknowns are theoretically generated and relate to one another. For example, random effects models typically assume random effects are normally distributed. This normality not only simplifies expressions and estimation procedures, but states that the DGP is hypothesized to generate parameters that are approximately normally distributed. Although theory may not explicitly dictate a particular shape of random effects, a typical random effects model is nevertheless estimated as though normality is explicitly assumed. Alternatively, theory may dictate non-normal distributions of random effects, and the normality assumption could be abandoned. When no explicit assumption is made about distributional shape, assuming a less constrained distribution may yield better estimates. Convenience assumptions should be second to theoretical assumptions, and should theory have no explicit assumption, then less constrained assumptions could be employed. In essence, assumptions are explicit statements about the theoretical process through which data are realized.

It is this second purpose that we believe is ignored in favor of the practical purpose of assumptions. Yet, it is from these model assumptions and consequential estimates that researchers derive predictions from and inferences about the DGP. Normality is often treated as an atheoretical goal that enables the use of classical inferential methods. Although these methods are robust to normality violations, the inferences are about a normal process from which data may not be generated. In the case of skewed data, achieving normality (instead of assuming skewness) is often pursued through data transformations.

\section{Data Transformations}

Observing skewed and non-normal data is a common occurrence. In a procrustean manner, these data are often distorted, censored, or truncated to impose normality upon one's data, rather than respecting the natural state of the observed data. Reaction time data is commonly skewed, as individuals' reaction times can easily be longer but not easily shorter. Likert and other constrained data are commonly skewed due to participants responding near boundaries. To meet normality assumptions, active areas of research encourage non-linear transformations, such as the arcsin, log, square root, or the ubiquitous Box-Cox procedure (of which $\log$ and root transforms are special cases). Although these procedures can achieve normality, many potential issues arise from such distortion.

The Box-Cox transform (Box \& Cox, 1964) is restricted to positive values, and its special cases - log and root - moreover only address positive skew. Transforming negative data requires positive translation by some random value subject to sampling error. To address negative skew with log and root transforms, data must further be reflected about some random variate that is subject to sampling error. The choice of which transformation and constants to use introduces data-dependent flexibility in analyses, which is unaccounted for in typical inferential techniques (Simmons, Nelson, \& Simonsohn, 2011; Gelman \& Loken, 2013). Moreover, the sampling error of the constants is ignored in inferential tests.

Importantly, transforming prior to analysis meaningfully alters inference. For example, a log transformed variable must be interpreted on a multiplicative scale rather than an additive scale; e.g., group differences must be interpreted as multiplicative differences. Means are no longer arithmetic expected values, but geometric means. The inference may even be reversed, such that if on the raw scale the mean of 


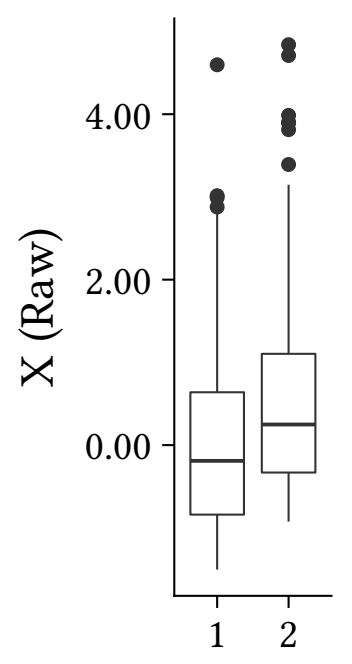

Group

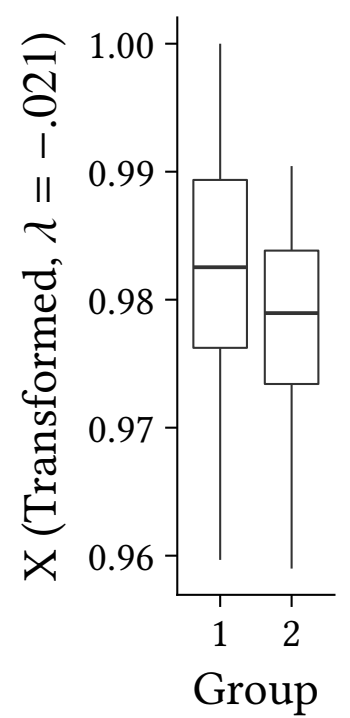

Figure 1. Left: Example of sign switching following a Box-Cox transformation. Right: Example of regression coefficient bias following a Box-Cox transformation. 5000 replicate datasets of $N=300$ were generated under the DGP: $y=.5 x+e$, $x \sim \operatorname{Normal}(0,1), e \sim \operatorname{Skew}-\operatorname{Normal}(\mu=0, \sigma=1, \alpha=8)$. Following a Box-Cox transformed regression, coefficients were transformed to the original scale. The solid vertical line is the true coefficient. The dashed vertical line is the mean inverse-transformed coefficient estimate. The mean coefficient was .565 , with a relative bias of .13 . The mean coefficient of a Skew-Normal regression model was .5001, with a relative bias of .0002 .

group 1 is greater than the mean of group 2, but on a transformed scale the opposite may be observed (see Figure 1). Although one can invert the transformation, this can bias predictions and estimates - By definition, the expected value of the inverse of a non-linear transform is not equivalent to the inverse non-linear transform of an expected value. Thus, estimating a coefficient by reversing the transformation produces a systematically biased estimate (see Figure 1).

Furthermore, when data or model complexities arise, the implications or process for transforming are unclear. Data from several groups can vary in skewness. Because transforms act on the unconditional variable, the data of any given group may remain or become skewed. Splitting the data by group, transforming, and combining the data modifies the expected values and scales for each group, potentially inflating or deflating differences between the groups. In the case of a latent variable or multilevel model, for example, how to normalize data, interpret coefficients, or evaluate predictive accuracy is difficult to determine. Finally, because some transformation procedures are model dependent (e.g., the Box-Cox) and operate on differing data vectors, engaging in model comparison is impossible without manually adjusting the likelihood.

In sum, transformations can be dangerous (Feng et al., 2014). While on the transformed scale inferences can be reversed, on the untransformed scale, estimates are biased. Because transformations involve data dependent decisions and randomly varying constants, they are subject to in- creased sampling error and researcher degrees of freedom unaccounted for in inferential tests (Gelman \& Loken, 2013; Simmons et al., 2011). Interpretation of the coefficients is meaningfully altered and unintuitive. Alternatively one may forgo the normality assumption and explicitly permit degrees of skewness or other perturbations to normality: Bayesian modeling offers a flexible framework for doing so.

\section{Bayesian Modeling}

$$
p(\theta \mid D)=\frac{\overbrace{p(D \mid \theta)}^{\text {Likelihood }} \overbrace{p(\theta)}^{\text {Prior }}}{\underbrace{\int p(D \mid \theta) p(\theta) d \theta}_{\text {Constant }}}
$$

Bayesian modeling is a coherent estimation and inferential framework in which a joint probability distribution is specified on the known quantities (data) and the unknown quantities (parameters) assumed to underlie the data generating process. Using Bayes' rule, one can compute from this joint probability distribution the posterior probabilities of parameters given the observations, $p(\theta \mid D)$, that are of interest to the researcher.

The joint probability consists of two specified components: The likelihood (the joint probability of the observations given the parameters) and the prior densities of the parameters. 
The likelihood represents the assumed distribution of observations given some parameters. For example, assuming observations are normally distributed about some point $\mu$ with variance $\sigma^{2}$ manifests in the use of a normal likelihood.

Priors encode information about the parameters irrespective of the data. Priors are multipurpose, and often misunderstood tools for improving estimation and inference. Regularizing priors combat unreasonable (e.g., not on the scale of the data) and boundary estimates (e.g., Lin, Gelman, Price, \& Krantz, 1999; Chung, Rabe-Hesketh, \& Choi, 2013; Chung, Rabe-hesketh, Gelman, Dorie, \& Liu, 2012; Gelman, 2006). Priors can provide probabilistic soft constraints that allow for model identification (Muthén \& Asparouhov, 2011). Empirical priors can be included to perform Bayesian updating. Functional priors (e.g., Horseshoe, Lasso; mixture priors; auxiliary variables) can guide inferential decisions and improve estimation efficiency (Carvalho, Polson, \& Scott, 2010; Bhadra, Datta, Polson, \& Willard, 2015; Park \& Casella, 2008).Hierarchical priors permit parameter estimates to share information or model a parameter process (e.g., hierarchical modeling).

Because Bayesian model assumptions are freely specified, it is very flexible and robust. Instead of transforming data to meet model assumptions, the latter can freely be altered to include characteristics of the data, and to model a DGP that can feasibly generate observations. Distributional assumptions for observations or unknown quantities can be included at will. Furthermore, it is well known that Bayesian model estimates and decisions can maintain frequentist properties, such as unbiasedness, interval coverage, and error rates (Box, 1980; Woodroofe, 1976; Diaconis \& Freedman, 1986; Little, 2006; Severini, 1993; Thatcher, 1964; Nicolaou, 1993; Berger et al., 1997; Peers, 1968). Importantly, regardless of assumptions, the inferential procedure remains the same in that inferences are drawn from the posterior distribution instead of a hypothetical sampling distribution.

\section{Skew-Normal Distribution}

We argue than when data are non-normally distributed due to skewness, Bayesian modeling of non-normal processes is powerful and robust. An assortment of distributions assume or permit skewness (Fernandez \& Steel, 1998; ArellanoValle, Del Pino, \& San Martín, 2002; Arellano-Valle \& Genton, 2005; Arellano-Valle \& Azzalini, 2013; Azzalini \& Capitanio, 1999; Azzalini, 1985; Azzalini \& Capitanio, 2003; Azzalini \& Dalla Valle, 1996), but among them is one that is intuitively appealing due to its addition of merely one parameter to a normal distribution, the Skew-Normal distribution:

$$
p\left(x_{i} \mid \xi, \omega, \alpha\right)=\frac{1}{\omega \pi} e^{\frac{\left(x_{i}-\xi\right)^{2}}{2 \omega^{2}}} \int_{\infty}^{\alpha\left(\frac{x_{i}-\xi}{\omega}\right)} e^{-\frac{t^{2}}{2}} d t
$$

The Skew-Normal is one of a family of skew distributions, and takes three parameters, a location, a scale, and a shape parameter. The shape parameter, $\alpha$, controls the skewness, where negative values indicate negative skew, zero indicates no skew, and positive values indicate positive skew. When the shape parameter is zero, the distribution is simply a normal distribution. Due to this property, the Skew-Normal can be essentially used in place of the normal distribution, and the normal distribution is merely a special case of the SkewNormal distribution in which the shape parameter is zero. As the shape parameter approaches infinity, the distribution is half-normal. The location and scale parameters, under the direct parameterization above, do not correspond to the mean and standard deviation, but they do affect the mean and standard deviation. To convert from these parameters to means and variances (Azzalini, 1985; Azzalini \& Dalla Valle, 1996), use the formulas: $\mu=\xi+\omega b \delta, \sigma^{2}=\omega^{2}\left(1-b^{2} \delta^{2}\right)$, where $b=\sqrt{\frac{2}{\pi}}, \delta=\frac{\alpha}{\sqrt{1+\alpha^{2}}}$.

Using the Skew-Normal likelihood in a Bayesian model frees one to no longer assume symmetric, normally distributed distributions of observations within groups, residuals from a linear model, latent abilities in latent variable models, or random effects in multilevel models. Unlike data transformations, the Skew-Normal distribution handles any real-valued negatively or positively skewed data, with normality as a special case. Compared to all the decisions and random variables required for data transformations, assuming data are distributed Skew-Normal results in fewer datadependent analytic decisions (Gelman \& Loken, 2013; Simmons et al., 2011).

Explicitly permitting skewness can increase the information gained about parameters of interest due to conditioning. The parameter contributing to non-normality constricts the range of plausible estimates of the parameters of theoretical interest. These parameters that cause normality perturbations are conditioned on when estimating other parameters, then marginalized out, resulting in more precise estimates of the parameters of interest (See Figure 2). Although the central limit theorem posits that sampling distributions of parameter estimates asymptotically approach normality as data increases regardless of the distribution, parameters of an ill-fitting distribution have less information than parameters of a well-fitting distribution, resulting in higher standard errors, decreased efficiency, and decreased power with the same number of observations. Moreover, an ill-fitting distribution imposes an untenable assumption about the DGP, and thus predictive utility will suffer.

The goal of the current paper is to assess the utility of the Skew-Normal distribution for asymmetric data analysis. We employ monte carlo simulation to demonstrate how models employing a Skew-Normal distribution can perform equally to or outperform normal-assumptive models in frequentist error rates, power, and bias, across an assortment of conditions with skewed or non-skewed data. 

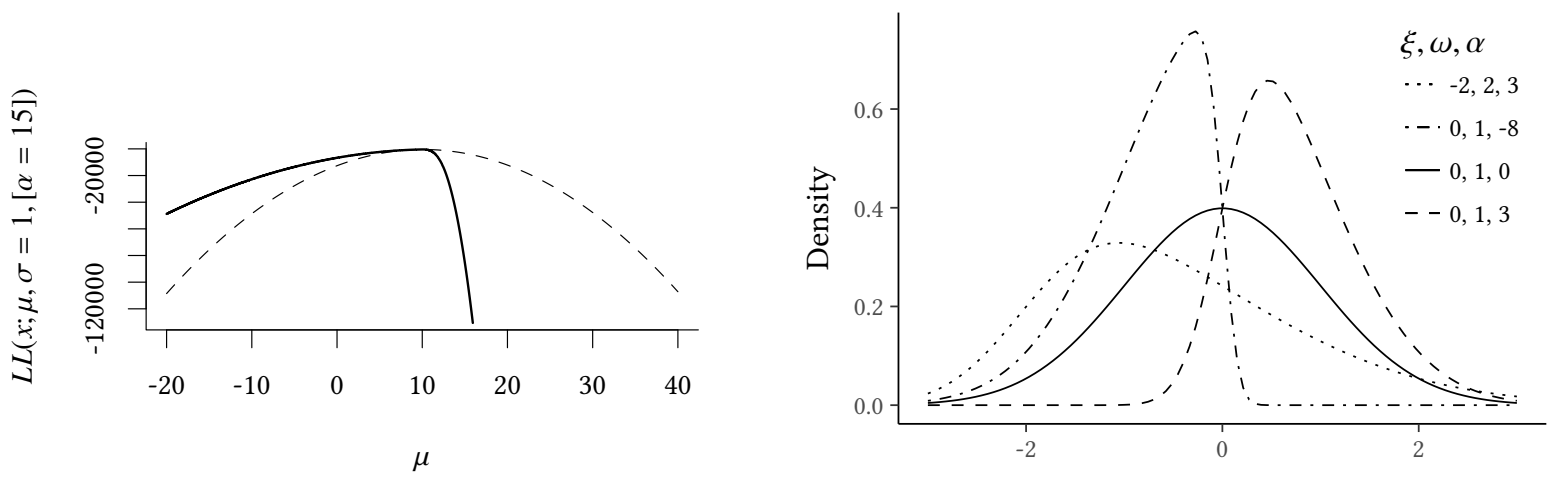

Figure 2. Left: Profile log-likelihood surface of a mean estimate under a Skew-Normal (solid) and Normal (dashed) model when $X \sim$ Skew-Normal. Information about parameters corresponds to the curvature of the log-likelihood at the optimal point. When data are skewed and skewness is modeled (solid), more information is available about $\mu$, resulting in lower standard error and greater power. Right: The Skew-Normal distribution under different parameters. $\xi, \omega, \alpha$ correspond to a location (not mean), scale (not SD), and skewness. Different values of these are plotted to demonstrate the shapes of data that the skew-normal can accommodate.

\section{Methods}

A monte carlo simulation of 120 conditions was performed to test the performance of five different approaches to skewed data. The hypothetical study is one in which there exist two experimental groups with differing means and an approximate effect size of either $\mathrm{d}=0$ or $\mathrm{d}=.4$, in order to assess type 1 error and power. The models therefore assess a difference between means of the two groups. The 120 conditions were made up of 5 sample sizes $(40,80,120,240,320) \mathrm{x}$ 4 skewnesses (alpha $=-8,0,3,8) \times 3$ group size equalities $\left(n_{1}=.25 N, n_{1}=.5 N, n_{1}=.75 N\right) \times 2$ Homoskedasticity conditions $\left(\omega_{2}=\omega_{1}, \omega_{2}=4 \omega_{1}\right)$.

For each condition, five analytic techniques were employed. The first is the Welch's $t$ statistic for mean differences that is robust to heteroskedasticity. The second transformed the data using the following $\log$ transformation on the outcome variable, $\mathrm{X}: X_{t}=\log (X-\min (X)+1)$, then used the Student's t-test to assess mean difference. The third used a Box-Cox transformation, $X_{t}=\frac{(X-\min (X)+1)^{\lambda}-1}{\lambda}$, the lambda parameter which was estimated per-sample using the boxcox function in the R MASS package (Venables \& Ripley, 2002). A student $t$-test was then employed to examine the mean difference between the groups.

The remaining two analytic techniques use the skewnormal distribution. One technique assumes homogenous variance, and the other does not. Both are linear models with a skew-normal likelihood function for the residuals, with a dummy-coded predictor indicating whether one is in group 2. Both models were estimated using Stan interfaced through the rstan package (Stan Development Team, 2016; Carpenter et al., 2017). For inferring whether 0 is a plausible estimate, we examined whether 0 was within the $95 \%$ posterior credible interval, akin to the use of confidence intervals for hypothesis testing in classical methods.

For each condition, we simulated 10,000 replicate datasets corresponding to the condition. Each analysis of each simulated dataset returned the estimated mean difference (on the untransformed scale), the standard error estimate (on the transformed scale, where applicable) or the posterior standard deviation of the estimate, a test statistic, a p-value (where applicable) and a 95\% posterior credible interval (where applicable). After 10,000 replicates were analyzed, we then computed the mean estimate, mean standard error (or posterior standard deviation), mean test statistic, and rejection rate (defined as the proportion of samples with $\mathrm{p}<.05$ or with posterior credible intervals excluding 0 ). Type 1 error, power, and relative bias of mean estimates were computed and presented below.

\section{Results}

\section{Type 1 Error}

Before considering whether skew-normal modeling indeed outperforms other transformation techniques, we must first establish that the models achieve acceptable nominal "Type 1" error rates (For Bayesian analyses, whether 0 was excluded as a plausible estimate). For the frequentist analyses, $\alpha$ was set to .05 , and for Bayesian analyses, $95 \%$ credible interval were employed. See Table 1 for incorrect rejection rates summarized across conditions.

When groups are homoskedastic, nominal error rates are achieved across all models. However, when groups are heteroskedastic, only the models that explicitly model or assume unequal variances achieve nominal error rates. That is, the Welch's t-test and the Bayesian Skew-Hetero model achieved the nominal error rates regardless of variance equal- 
Table 1

Type 1 error rates across $N$ conditions. Bolded entries represent the error rates across $N$, Skewness, and N ratio conditions.

\begin{tabular}{rrrrrrr}
\hline Variance Ratio & Skewness & Box-Cox & Log & Normal & Skew Hetero & Skew Homo \\
\hline $1: 1$ & -8 & .052 & .048 & .051 & .055 & .044 \\
$1: 1$ & 0 & .051 & .049 & .051 & .047 & .058 \\
$1: 1$ & 3 & .051 & .050 & .050 & .047 & .057 \\
$1: 1$ & 8 & .049 & .049 & .052 & .054 & .042 \\
\hline $1: 4$ & -8 & .272 & .199 & .054 & .057 & .839 \\
$1: 4$ & 0 & .072 & .292 & .049 & .047 & .195 \\
$1: 4$ & 3 & .107 & .358 & .052 & .049 & .641 \\
$1: 4$ & 8 & .232 & .448 & .053 & .056 & .838 \\
\hline Variance Ratio & $\mathrm{N} \mathrm{ratio}$ & Box-Cox & Log & Normal & Skew Hetero & Skew Homo \\
\hline $1: 1$ & $1: 3$ & .051 & .049 & .052 & .050 & .050 \\
$1: 1$ & $1: 1$ & .050 & .049 & .049 & .052 & .051 \\
$1: 1$ & $3: 1$ & .052 & .050 & .052 & .050 & .049 \\
\hline $1: 4$ & $1: 3$ & .050 & .105 & .050 & .051 & .527 \\
$1: 4$ & $1: 1$ & .168 & .345 & .051 & .054 & .645 \\
$1: 4$ & $3: 1$ & .294 & .523 & .055 & .052 & .713 \\
\hline $\mathbf{1 : 1}$ & & $\mathbf{. 0 5 1}$ & $\mathbf{. 0 4 9}$ & $\mathbf{. 0 5 1}$ & $\mathbf{. 0 5 1}$ & $\mathbf{. 0 5 0}$ \\
$\mathbf{1 : 4}$ & &. $\mathbf{1 7 1}$ & $\mathbf{. 3 2 4}$ & $\mathbf{. 0 5 2}$ & $\mathbf{. 0 5 2}$ & $\mathbf{. 6 2 8}$ \\
\hline
\end{tabular}

Table 2

Power across $N$ and group size conditions. Bolded entries represent the power across all N, Skewness, and N ratio conditions. Cells in which the corresponding nominal error rate was not achieved are omitted for clarity.

\begin{tabular}{rrrrrrr}
\hline Variance Ratio & Skewness & Box-Cox & Log & Normal & Skew Hetero & Skew Homo \\
\hline $1: 1$ & -8 & .621 & .482 & .550 & .558 & .737 \\
$1: 1$ & 0 & .553 & .536 & .548 & .527 & .557 \\
$1: 1$ & 3 & .594 & .594 & .549 & .535 & .627 \\
$1: 1$ & 8 & .643 & .629 & .552 & .557 & .734 \\
\hline $1: 4$ & -8 & - & - & .578 & .544 & - \\
$1: 4$ & 0 & - & - & .567 & .559 & - \\
$1: 4$ & 3 & - & - & .561 & .593 & - \\
$1: 4$ & 8 & - & - & .558 & .637 & - \\
\hline Variance Ratio & N ratio & Box-Cox & Log & Normal & Skew Hetero & Skew Homo \\
\hline $1: 1$ & $1: 3$ & .579 & .547 & .522 & .504 & .641 \\
$1: 1$ & $1: 1$ & .659 & .617 & .612 & .607 & .715 \\
$1: 1$ & $3: 1$ & .571 & .517 & .516 & .521 & .635 \\
\hline $1: 4$ & $1: 3$ & - & - & .694 & .699 & - \\
$1: 4$ & $1: 1$ & - & - & .606 & .626 & - \\
$1: 4$ & $3: 1$ & - & - & .398 & .425 & - \\
\hline $\mathbf{1 : 1}$ & &. $\mathbf{6 0 3}$ &. $\mathbf{5 6 0}$ &. $\mathbf{5 5 0}$ & $\mathbf{. 5 4 4}$ & $\mathbf{. 6 6 4}$ \\
$\mathbf{1 : 4}$ & & - & - & .566 & .583 & - \\
\hline
\end{tabular}


Table 3

Relative Bias of estimated mean differences across group size conditions.

\begin{tabular}{rrrrrrr}
\hline Variance Ratio & Skewness & Box-Cox & Log & Normal & Skew Hetero & Skew Homo \\
\hline $1: 1$ & -8 & -0.025 & 0.026 & 0.001 & -0.001 & 0.001 \\
$1: 1$ & 0 & -0.006 & 0.044 & -0.000 & -0.001 & -0.002 \\
$1: 1$ & 3 & 0.024 & 0.034 & -0.001 & 0.000 & -0.000 \\
$1: 1$ & 8 & 0.044 & 0.043 & 0.002 & 0.001 & -0.001 \\
\hline $1: 4$ & -8 & 0.311 & -0.754 & -0.003 & -0.052 & 2.003 \\
$1: 4$ & 0 & -0.080 & -0.850 & 0.001 & -0.001 & -0.008 \\
$1: 4$ & 3 & -0.425 & -0.769 & -0.000 & 0.030 & -1.394 \\
$1: 4$ & 8 & -0.667 & -0.802 & -0.000 & 0.055 & -2.006 \\
\hline
\end{tabular}

ity. Neither Box-Cox, Log, nor Skew-Homogenous models achieved nominal error rates when variances were unequal. For the Skew-Homogenous model, this is to be expected. When heteroskedastic data is modeled without permitting heteroskedasticity, the Skew-Homogenous model cannot by definition have the proper expected values due to the expectation being in part defined by a scale parameter, and this results in spurious effects when the true effect is zero, and incorrect effect estimates when an effect exists. Nevertheless, when the model assumptions match the DGP, type 1 error is maintained across the Skew-Normal models.

\section{Power}

Power was assessed for each condition and each model by computing the proportion of replicates for which 0 was rejected as a plausible estimate when a true effect is present. Again, for non-Bayesian models, $\alpha$ is set to .05, with the null hypothesis being rejected if $\mathrm{p}<.05$. For Bayesian models, 0 was excluded as a plausible estimate if 0 is outside the $95 \%$ credible intervals. See figure 3 and Table 2 for results.

When variances are equal and skew is present, the Skew-Homogenous model very obviously outperforms the other models. Compared to the Welch t-test, the SkewHomogenous model was $15 \%$ more powerful when skew was present, on average. Compared to Box-Cox transformed models, the Skew-Homogenous model was $8 \%$ more powerful when skew was present, on average. Importantly, when no skew was present, the Skew-Homogenous model was no less powerful than any other technique.

As would be expected given the Type-1 error, the "power" of the Skew-Homogenous model when variances are unequal can be artificially greater than that of other models. Ironically, in some conditions, the probability of falsely rejecting the null when the null is true exceeds the probability of correctly rejecting the null when the null is false. The sheer magnitude of this inflation of errors suggests that researchers employing a Skew-Normal model should take great care in assuring that assumptions of the model adequately match their own data, though this is apparently true of the Box-Cox and Log models as well. In this particular case, the variances differ so strongly, it should be of no surprise that any model failing to account for heterogenous variances performed relatively poorly.

When variances were unequal, the Skew-Heterogenous performed essentially as well as the Welch's t-test. Although a small and unexpected difference, the Skew-Heterogenous model exhibited less power when skew was negative than when skew was positive, compared to the Welch test.

In sum, when variances were homogenous, the SkewHomogenous model consistently exhibited greater power than the Skew-Heterogenous model, the Welch's t-test, and normality-assumptive models with data transformations. When variances were heterogenous, the Skew-Hetero model and the Welch's t-test performed similarly. Whether these models' power is greater or lesser than the other models is irrelevant, only because the other models' Type 1 error rate were vastly incorrect due to the failure to account for heteroskedasticity.

\section{Relative Bias}

Relative bias was computed as:

$$
\text { Relative Bias }=\frac{\left(\hat{\mu}_{2}-\hat{\mu}_{1}\right)-\left(\mu_{2}-\mu_{1}\right)}{\left(\mu_{2}-\mu_{1}\right)}
$$

, with 0 being the ideal estimate. Relative bias estimates are provided in Table 3. For the Box-Cox and Log models, the bias is reported on the original scale, not the transformed scale. These are expected to be biased due to the fact that $f^{\prime}(E(x)) \neq E\left(f^{\prime}(x)\right)$, where $f^{\prime}$ is some inverse of a nonlinear function (e.g., the inverses of the Box-Cox or Log transforms, both non-linear transforms).

As one can see in Table 3, the Box-Cox and Log models are, as expected, consistently biased, with the Box-Cox model only demonstrating minimal bias when data are not skewed and variances are homogenous. However, the bias is relatively small when variances are homogenous. The SkewHomogenous model, Skew-Heterogenous, and Welch models all demonstrate no bias when variances are homogenous. 


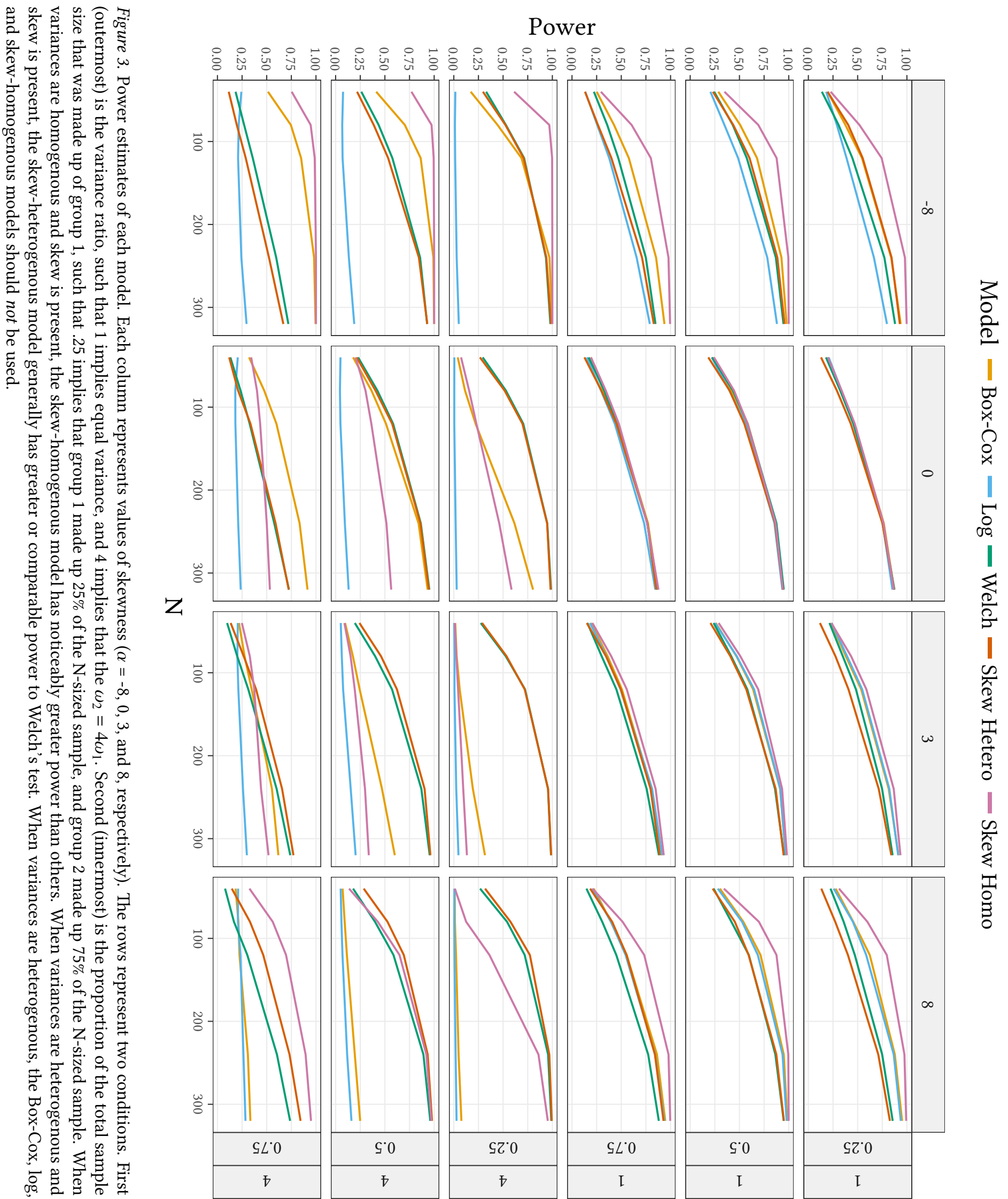


However, when variances are heterogenous, the BoxCox, Log, and in particular the Skew-Homogenous models all demonstrate substantial bias. The Welch's mean estimates do not demonstrate bias as one may expect, given that the Welch's t-test only requires a raw mean difference and variance estimates. The Skew-Heterogenous model does demonstrate some slight bias, although small. The SkewHeterogenous model is a seemingly new method for modeling skewed variates with differing scale parameters. This model in particular could be improved in the future to further decrease the slight bias in the estimates.

\section{Summary}

Type 1 error, power, and relative bias were assessed for the Welch's t-test, Box-Cox transformed t-test, log transformed ttest, and two skew-normal models differing only in whether homogeneity of variance is assumed. When the analytic assumptions match the data generating process, type 1 error is maintained, power is greater, and relative bias is negligible or nonexistent. Namely, if variances are equal and skew is present, the skew-homogenous model exhibits the greatest power, type 1 error control, and no bias. If variances are unequal and skew is present, the skew-heterogenous model demonstrates power nearly equal to the Welch's t-test, type 1 error control, and negligible bias.

Moreover, because the normal distribution is merely a special case of a skew-normal distribution, assuming a skewnormal distribution is a fairly safe default assumption. When no skew is present, the skew-normal models (when assumptions are met) retained their type 1 error control, power, and relative bias properties, relative to the normal model.

\section{Predictive Utility}

Type 1 error, power, and bias are not the only concerns to scientists. For a model to be useful, the model should demonstrate strong predictive performance. Two popular methods in contemporary Bayesian analyses for assessing model performance are to perform posterior predictive checking and to estimate the leave-one-out (LOO) predictive performance. We will use these methods to assess the normal-assumptive model, Box-Cox, and the two skew-normal models. The log model is excluded due to its generally poor performance in the above simulations, and because the log transformation is a special case of the Box-Cox transformation.

The posterior predictive check (PPC) can take many forms, but an intuitively appealing method is to simply generate model-implied datasets and visually examine the extent to which replicate model-implied datasets resemble the observed data (Gelman, 2003). If a model sufficiently approximates the data generating process, the model-implied replicate datasets should closely resemble the observed data. Generating replicate datasets is as simple as obtaining point parameter estimates and plugging those point estimates into a generating function (such as a random normal deviate generator). However, doing so does not incorporate uncertainty in the point estimate. Instead, posterior predictive datasets are obtained by marginalizing over the posterior of the parameter estimates from a Bayesian model. That is, instead of plugging in point estimates, the posterior distribution of estimates, $p(\theta \mid D)$, is used to obtain a posterior predictive distribution, $p\left(D_{\text {rep }} \mid D\right)$ :

$$
p\left(D_{\text {rep }} \mid D\right)=\int p\left(D_{\text {rep }} \mid \theta\right) p(\theta \mid D) d \theta
$$

We will use the latter method to assess the extent to which generated model-produced datasets of these models match the observed dataset.

The leave-one-out prediction error can be estimating using the LOOIC (Vehtari, Gelman, \& Gabry, 2015). LOOIC can be used for model comparison, such that the model with the lowest LOOIC should perform better than the rest at out of sample prediction. Additionally, the contribution of each individual to the prediction error can be computed, allowing examination into where models are failing to adequately predict observed cases. We will use the LOOIC and PPC to compare the models above, with some modification. Two datasets with $n_{1}=150, n_{2}=150$, positive skew $(\alpha=8)$, and a standardized mean difference of roughly $d=.4$ was created, with the only difference being that one dataset has homogenous and the other heterogenous group variances, akin to the data used in the simulations. Namely, we will convert the non-Bayesian models to their Bayesian equivalents, permitting us to conduct a PPC and compute the LOOIC for each model for a given dataset.

Models of different data transformations cannot be, without adjustments, be compared with respect to the loglikelihoods. The LOOIC utilizes the log-likelihoods for comparison, and thus the log-likelihoods must be adjusted. In order to adjust the likelihoods, we multiplied the likelihood of each observation by the absolute determinant of the Jacobian of the transformation applied to the data. In the case of the Box-Cox transformation, we multiply by $\prod_{i} y_{i}^{(\lambda-1)}$ if $\lambda \neq 0$, and by $\prod_{i} \frac{1}{y_{i}}$ if $\lambda=0$ (these adjustments were computed on the log scale and added to the log-likelihood).

Posterior Predictive Checks. Figures 4 show the observed variables along with several model-implied posterior predicted datasets for homogenous and heterogenous datasets, respectively.

Posterior predictive "p-values" (PPC p-values; Gelman, 2003) were computed at each percentile ranging from .01 to 99. PPC p-values represent the proportion of model-implied replicate datasets for which some sample statistic is greater than the observed sample statistic. These $\mathrm{p}$-values can be interpreted as the extent of misfit between observed data and the model-implied predictive data, such that an extreme $\mathrm{p}$ value suggests that the observed sample statistic is unlikely 

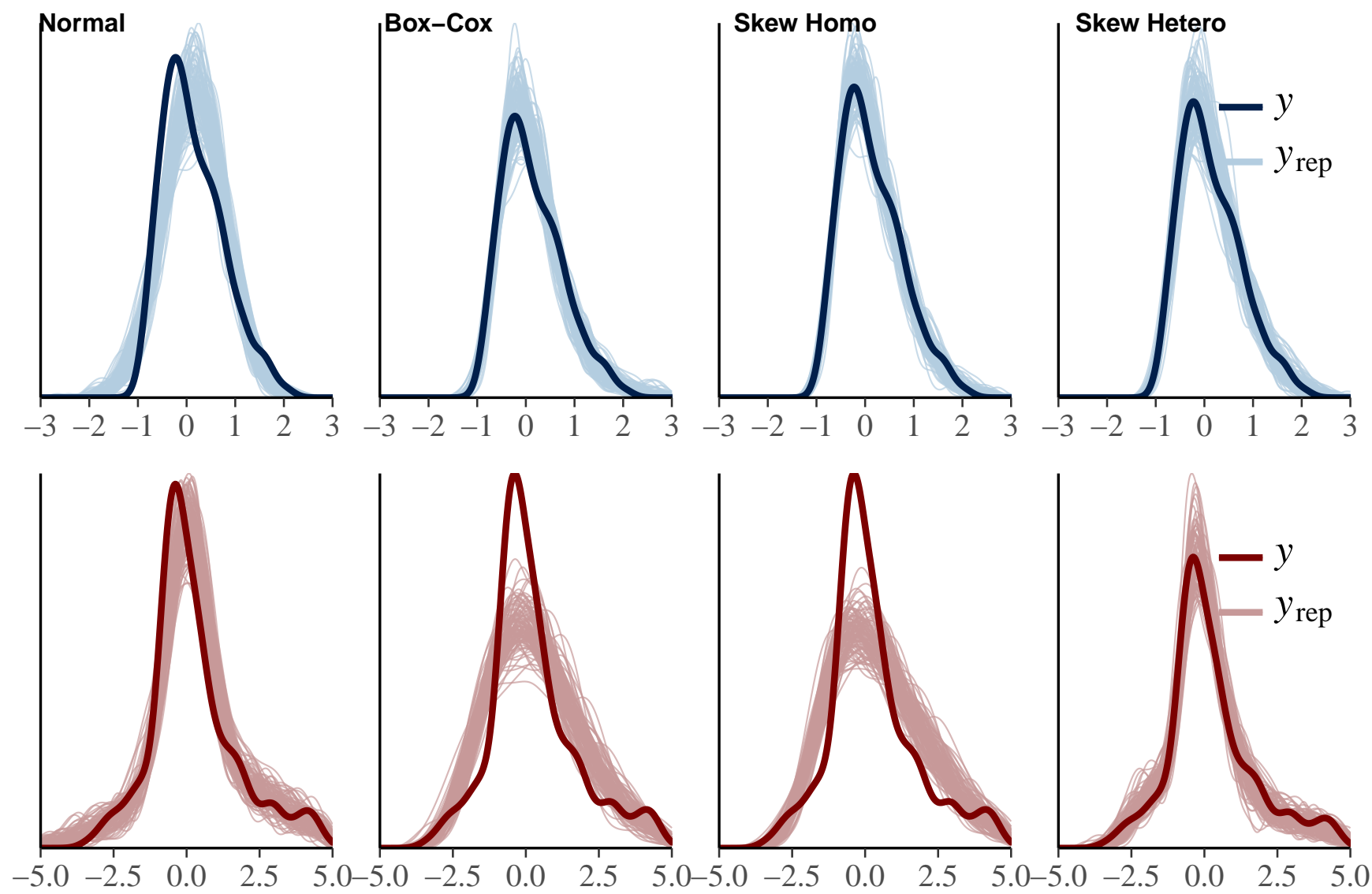

Figure 4. Posterior predictive model-implied datasets of each model. The bold line represents the observed distribution of data. The top row (blue) corresponds to homoskedastic data, and the bottom row (red) heteroskedastic data. When variances are homogenous, all but the normal model fit the observed data well. When variances are heterogenous, the skew-heterogenous model best fits the observed data.

given the model implications. For each replicate dataset, we performed a logspline density estimation for interpolation, and from this density computed the quantiles corresponding to each percentile $\in\{.01, .02, \ldots, .98, .99\}$. We then computed the proportion of replicate datasets' quantiles that exceeded the observed quantile, at each percentile. Ideally, the PPC p-value should equal .5 at each percentile, suggesting that $50 \%$ of model-implied datasets' quantiles lie above (and thus also below) the observed quantile. The sum of "extreme" PPC p-values (PPC p-value $<.05$ or $>.95$ ) is computed for each model. These sums and a subset of the PPC $\mathrm{p}$-values are presented in Table 4.

In the case of homogenous variances, the normalassumptive model mismatches the observed data. The mode is overestimated, and observations near the tails are underestimated. Data near the median are overestimated consistently. In contrast, Box-Cox and the skew-normal models perform well and similarly on the homogenous data.

In the case of heterogenous variances, the normalassumptive model again mismatches the observed data in a manner similar to the case of homogenous variance. However, the Box-Cox and skew-homogenous models drastically mismatch the data. They both underestimate and overestimate near the left and right tails, respectively, and underestimate the number of observations near the observed mean. All models, except for the skew-heterogenous model, severely overestimate the median. The skew-heterogenous model replicates the observed data very well.

LOOIC and pointwise LOOIC. The LOOIC estimates of each model under homogenous and heterogenous variance scenarios are provided in Table 5. As expected, when variances are homogenous, the skew-homogenous and skewheterogenous models have the lowest LOOIC, and thus the lowest leave-one-out predictive error. When variances are heterogenous, the skew-heterogenous model has the lowest LOOIC, followed by the heteroskedastic normal model.

A useful feature of the LOOIC is the ability to examine how observations contribute to the LOO error estimate. Figure 5 plots the performance of each model in predicting each observation. Under homogenous variances, the skew models 
Table 4

Posterior predictive p-values across a selection of percentiles. Extreme p-values $(<.05,>.95)$ suggest that the model-implied replicate datasets noticeably mismatch the observed data at the same percentile. A p-value of .5 is ideal. The bottom row provides the number of quantiles (out of 99) for which the PPC p-value was extreme. The lower this sum, the better the observed data match the model-implied replicate datasets across the entire distribution. N: Heteroskedastic Normal, BC: Box-Cox, S-Ho: SkewHomogenous, S-He: Skew-Heterogenous.

\begin{tabular}{rrrrrrrrr}
\hline & \multicolumn{4}{c}{ Homogenous } & \multicolumn{3}{c}{ Heterogenous } \\
\cline { 2 - 10 } \%tile & $\mathrm{N}$ & BC & S-Ho & S-He & $\mathrm{N}$ & BC & S-Ho & S-He \\
\hline 01 & 0.00 & 0.05 & 0.16 & 0.16 & 0.03 & 0.61 & 0.84 & 0.59 \\
05 & 0.01 & 0.59 & 0.74 & 0.73 & 0.31 & 0.60 & 0.87 & 0.61 \\
10 & 0.26 & 0.82 & 0.83 & 0.83 & 0.18 & 0.01 & 0.03 & 0.27 \\
20 & 0.79 & 0.80 & 0.69 & 0.70 & 0.42 & 0.01 & 0.02 & 0.39 \\
30 & 0.92 & 0.68 & 0.52 & 0.53 & 0.85 & 0.18 & 0.28 & 0.56 \\
40 & 0.95 & 0.54 & 0.40 & 0.41 & 0.98 & 0.69 & 0.82 & 0.76 \\
50 & 0.95 & 0.41 & 0.35 & 0.36 & 0.99 & 0.94 & 0.98 & 0.78 \\
60 & 0.92 & 0.32 & 0.34 & 0.37 & 0.95 & 0.95 & 0.99 & 0.51 \\
70 & 0.82 & 0.28 & 0.37 & 0.38 & 0.68 & 0.93 & 0.99 & 0.33 \\
80 & 0.58 & 0.32 & 0.41 & 0.43 & 0.47 & 0.83 & 0.96 & 0.31 \\
90 & 0.19 & 0.48 & 0.48 & 0.51 & 0.48 & 0.48 & 0.79 & 0.47 \\
95 & 0.08 & 0.63 & 0.56 & 0.60 & 0.36 & 0.24 & 0.49 & 0.56 \\
99 & 0.07 & 0.79 & 0.65 & 0.68 & 0.14 & 0.12 & 0.22 & 0.58 \\
\hline & 18 & 0 & 0 & 0 & 26 & 27 & 49 & 0 \\
\hline
\end{tabular}

Table 5

LOOIC estimates. The lower the LOOIC, the lower the LOO predictive error, and thus the greater predictive utility.

\begin{tabular}{rrrrr}
\hline & \multicolumn{2}{c}{ Homogenous } & \multicolumn{2}{c}{ Heterogenous } \\
\cline { 2 - 5 } & LOOIC & SE $_{\text {LOOIC }}$ & LOOIC & SE $_{\text {LOOIC }}$ \\
\hline Skew Homo & 503.99 & 23.59 & 1132.29 & 34.02 \\
Skew Hetero & 505.99 & 23.55 & 921.80 & 33.37 \\
Box-Cox & 518.48 & 24.49 & 1137.84 & 32.79 \\
Normal Hetero & 559.11 & 25.57 & 976.07 & 33.95 \\
\hline
\end{tabular}

perform similarly across all observations - Their respective points and lines overlap considerably. Both skew models and Box-Cox tend to perform better at the skewed tails of the observations than the normal-assumptive model.

When variances are heterogenous, the skew heterogenous model demonstrates better predictive performance than the other models. Compared to the heteroskedastic normal model, the skew heterogenous model better predicts the skewed tail and modal region of the observations. This is perhaps the most important comparison for the heterogenous data, given that the other models exhibited substantial bias under the same conditions across the above simulated datasets. The Box-Cox and skew homogenous models generally performed poorly when predicting those in group 1 . For those in group 2, these two models fared better, though were still noticeably worse than the normal or skew heterogenous models in all regions but the immediate modal region of observations.
Summary. In sum, the best LOO predictive performance occurs when a model accurately matches the data generating process. In this example with two groups with skewed data and equal variances, a model which assumes group data may be skewed and have equal variances exhibits the best fit, measured by visual posterior predictive checks, posterior predictive $\mathrm{p}$-values, and leave-one-out prediction error. Likewise, when two groups with skewed data and unequal variances are observed, a model which assumes group data may be skewed and have unequal variances exhibits the best fit, measured by visual PPC, PPC p-values, and leaveone-out prediction error.

\section{Motivating Example}

We now turn to an example of analyzing real data using the skew-normal distribution. The data in question originate from an unpublished study by Martin and Masicampo (2013). 


\section{Model $\leftarrow$ Box-Cox $\leftarrow$ Normal $\leftarrow$ Skew Hetero $\leftarrow$ Skew Homo}
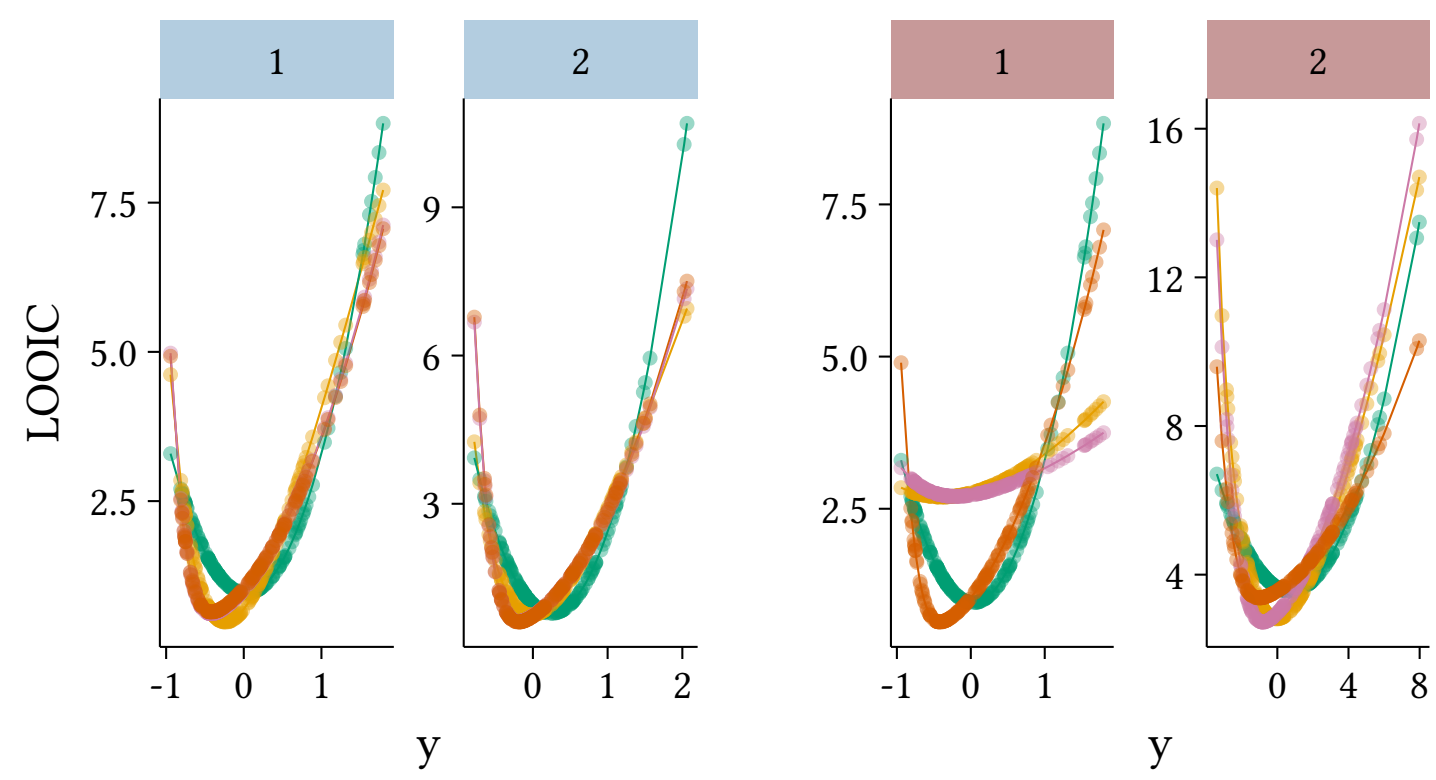

Figure 5. Pointwise LOOIC contribution for each model. On the x-axis is the observation. On the $\mathrm{y}$-axis is the amount the observation contributed to the LOO prediction error (lower is better). Panels 1 and 2 represent those in group 1 and 2 , respectively. The left two panels (blue) correspond to homoskedastic data, and the right two (red) to heteroskedastic data.

The study is a 2 (Target: Science, Religion) $\times 2$ (Pair: Bad, Good) factorial experiment with reaction time as the dependent variable. The parameter of primary interest is the interaction between target and pair. Instead of a typical normalityassumptive ANOVA, we will employ a linear model with a skew-normal assumption that permits heterogenous variance and skew.

$$
\begin{aligned}
& \widehat{\mathrm{RT}}_{i}=\overbrace{\beta_{1}}^{\text {Intercept }}+\overbrace{\beta_{2} X_{1 i}}^{\text {Target }}+\overbrace{\beta_{3} X_{2 i}}^{\text {Pair }}+\overbrace{\beta_{4} X_{1 i} X_{2 i}}^{\text {Interaction }}\left(\bar{y}_{i} \text { prediction }\right) \\
& \log \sigma_{i}=\gamma_{1}+\gamma_{2} X_{1 i}+\gamma_{3} X_{2 i}+\gamma_{4} X_{1 i} X_{2 i} \quad \text { (SD prediction) } \\
& \alpha_{i}=\zeta_{1}+\zeta_{2} X_{1 i}+\zeta_{3} X_{2 i}+\zeta_{4} X_{1 i} X_{2 i} \quad \text { (Skew prediction) } \\
& \mathrm{RT}_{i} \sim \mathrm{SN}\left(\xi_{i}, \omega_{i}, \alpha_{i}\right) \\
& \text { (Likelihood) }
\end{aligned}
$$

where $\xi_{i}, \omega_{i}$ are computed from the following equations:

$$
\begin{aligned}
b & =\sqrt{\frac{2}{\pi}} \\
\delta_{i} & =\frac{\alpha_{i}}{\sqrt{1+\alpha_{i}^{2}}} \\
\omega_{i} & =\frac{\sigma_{i}}{\sqrt{1-\delta_{i}^{2} b^{2}}} \\
\xi_{i} & =\widehat{\mathrm{RT}_{i}}-\omega_{i} \delta_{i} b
\end{aligned}
$$

Reaction time was regressed onto effect-coded Pair (Good $=1$, $\mathrm{Bad}=-1)$, Target $($ Religion $=1$, Science $=-1$ ), and their interaction, with a skew-normal residual distribution. To permit residual variance and skew to vary by condition, the $\log ^{1}$ standard deviation and alpha parameters are predicted by conditions. The data were filtered to remove $\mathrm{RT}<300 \mathrm{~ms}$ and $>2000 \mathrm{~ms}$, assuming that responses too quick or too slow originate from a separate DGP of interest (e.g., mistaken key presses, guessing, distraction). After removing incorrect responses for the sake of simplicity, this procedure left 254 observations. Minimally informative priors were employed for the RT and variance predictive coefficients. Because the skew-normal approaches a half-normal in the limit as $|\alpha| \rightarrow \infty$ with little identifiable difference between large $\alpha$ values, an identifying prior was placed on the $\alpha$ predictors. Annotated R code for estimating this model in rstan (Stan Development Team, 2016) and brms (Bürkner, in press) is available in appendix A, with the stan model code in appendix B.

Model fit is assessed first through a visual PPC (See Figure 6, top left pane). The posterior predictive data aligns well with the observed distribution of data, and we are

\footnotetext{
${ }^{1} \sigma_{i}$ is predicted on a log scale to allow for a linear regression on an unconstrained real line.
} 

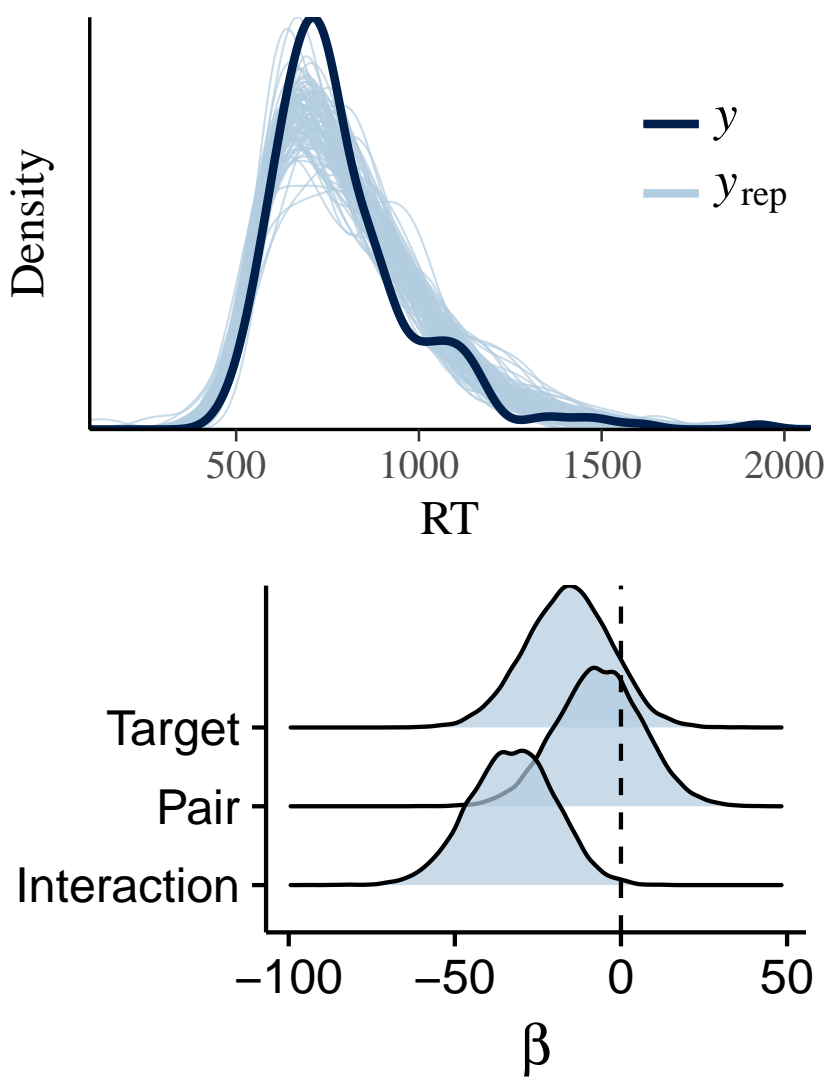
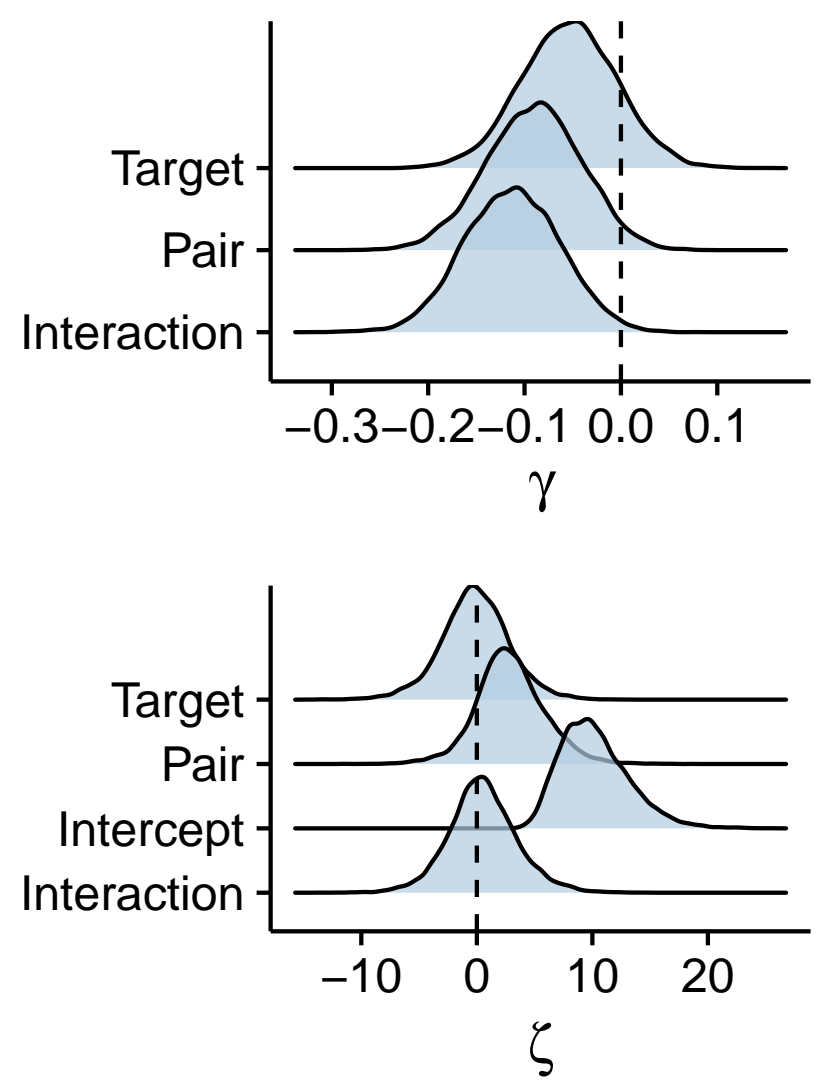

Figure 6. The posterior predictive distribution of reaction times (Top left). The posterior distributions for each of the $\beta$, $\gamma$, and $\zeta$ coefficients are in the bottom left, top right, and bottom right respectively. The intercepts for $\beta$ and $\gamma$ are excluded for space. Residuals are notably skewed on average $-\zeta_{1}$ is large and positive - but the conditions vary little in skewness. $E\left(\gamma_{1}\right)=5.27$, with the conditions plausibly varying from one another, suggesting heteroskedasticity is present. The posterior for $\beta_{4}$ (the interaction effect) suggests that the interaction effect is most probably negative.

therefore comfortable with the assumptions made about the DGP. Second, the posterior marginal distribution of the interaction coefficient (See Figure 6, bottom left pane) is described by the expected a-posteriori estimate (EAP), posterior standard deviation, and the $95 \%$ highest density interval (HDI), $\hat{\beta}_{4}=-32.797, \sigma_{\hat{\beta}_{4}}=12.87,95 \%$ HDI $[-57.38,-7.54]$. The posterior probability that the interaction is negative $(P P N=1-P P P)$ is .9958 . A Bayesian t-like statistic can be computed as $\frac{\hat{\beta}_{4}}{\sigma_{\hat{\beta}_{4}}}=-2.547$. Finally, for ease of interpretation, an approximate standardized effect size for the effect-coded interaction can be computed as

$$
d_{1(1)}-d_{1(-1)}=\frac{4 \beta_{4}}{\bar{\sigma}}=-.671[-1.19,-.18], \sigma_{d-d}=.26
$$

, where $\bar{\sigma}$ is the average standard deviation across conditions $\left(\bar{\sigma}=e^{\gamma_{1}}\right)$, which suggests that the approximate $\mathrm{d}$ statistic for the difference in RT between good and bad pairings is .87 lower when the target is religion (i.e., responses were much quicker to respond to religion-good than science-good pair- ings). From this wealth of information, we can infer that the direction of the interaction is negative with high probability, and the inner 95\% most plausible estimates suggest that the interaction is expected to be fairly large.

For comparison, a similar heteroskedastic normal model was estimated. The interaction coefficient was estimated with less certainty, $\hat{\beta}_{4}=-26.08, \sigma_{\hat{\beta}_{4}}=12.95,95 \%$ HDI $[-50.06$, $.32]$, ppn $=.9785, " \mathrm{t} "=-2.014, d_{\text {inxn }}=-.522[-1.00, .01]$. The LOOIC for the normal model (3423.42) was worse than for the skew-normal model (3354.04).

\section{Summary and Suggestions}

A complex Skew-Normal model of reaction time data was fit such that each group was assumed to be distributed SkewNormally with different mean, variance, and skewness parameters. From this model, the posterior probability of an an interaction was computed, and suggested that a-posteriori we should assume that the interaction is negative, such that 
responses were faster when religion is paired with good than when science is paired with good. Moreover, the SN model exhibited better predictive performance than a similarly flexible Normal model, the model-implied posterior predictive datasets of the skew-normal model better matched the observed data, and the estimate of interest was more precise under the skew-normal model.

The model was estimated using Bayesian modeling through the R interface for Stan (see Appendices A and B; Stan Development Team, 2016). Stan is an incredibly flexible statistical tool for sampling from posteriors through MCMC (using NUTS or HMC), for approximating posteriors through variational Bayes, and for optimization (Carpenter et al., 2017). So long as the researcher can express a model through distributions on observations, unknowns, and parameters, Stan can estimate posterior distributions for an enormous class of models with as many or as few assumptions about the DGP as the modeler desires. Stan further allows one to compute and save model-implied datasets and log likelihoods of each observation within each variable for use in model comparison and model checking. We recommend Stan through RStan for researchers who want complete control over the model structure and assumptions.

A relatively new $\mathrm{R}$ package, brms, is available and is a user-friendly package for generating Stan code (Bürkner, in press). The brms package requires that the user specify a series of linear models on the data or parameters of interest, and includes enormous flexibility. It includes, presently, 34 likelihoods (including the Skew-Normal), along with the ability to estimate measurement error models, weighted regression models, meta-analytic models, random effects models, hurdle models, and mixture models, or any combination therein, along with handling censored or truncated data, the ability to employ horseshoe or lasso methods for shrinkage, and spline models. Brms automatically converts from $\xi, \omega$ to $\mu, \sigma$ as well, allowing users of the skew-normal model to interpret the predictions as means and the residual scale as a residual standard deviation, just as in normal linear models. Brms can also compute LOOIC, conduct posterior predictive checks, and approximate Bayes factors, if desired. Because linear models on parameters can be estimated as well as observations, the analyses conducted in this paper can be replicated by instructing brms to estimate an effect-coded linear regression with skew-normal residuals, and simultaneously estimate a effect-coded linear regression model on the alpha and sigma parameters (See appendix A). We recommend brms for researchers who can express their model as a series of linear models on observed variables or parameters.

Finally, for those who wish to fit a skew-normal model with a maximum likelihood estimator and use frequentist inference, the $\mathrm{R}$ package sn permits linear models with skewnormal, skew-t, and skew-cauchy distributions (Azzalini, 2017). However, this package does not presently permit heterogeneous skew or variance, and the notion of posterior predictive checks is absent in the frequentist paradigm. Nevertheless, for those who wish to experiment with the skew-t and skew-cauchy distributions without manually implementing such distributions in Stan, the sn package is recommended.

\section{Discussion}

This paper aimed to assess the utility of the skew-normal distribution for fitting skewed data. The simulations suggest that adopting a Skew-Normal model and evaluating the posterior estimates in a manner analogous to classical statistics maintains type 1 error, exhibits negligible to no bias, and is at least as powerful as utilizing a normal-assumptive model. Moreover, when skew is not present, the Skew-Normal model again exhibits similar type 1 error, bias, and power as a normal-assumptive model, suggesting that the Skew-Normal model may be a relatively safe default.

Importantly, but unsurprisingly, models perform best in predictive utility and maintain frequentist properties when the model assumptions match the data generating process. Although distorting observations of the DGP via transformations to meet a normality assumption may be better than assuming normality and ignoring assumption violations, models which explicitly permit perturbations to normality can be more powerful, exhibit less bias, and more accurately predict observations. Moreover, distorting observations of the DGP for atheoretical reasons or computational convenience is questionable, because inference is conducted on a normal-assumptive process that can not tenably generate the observed data.

We make the recommendation that psychologists do their best to ensure that models best match the observations of a process, instead of ensuring the data best match the model. By this, we suggest that proposed models of any complexity should be able to feasibly generate the observed data, and only then should inferences be made about the true data generating process. This typically amounts to choosing as a likelihood a distribution that best reflects the observed distributions, or choosing a distribution for which other distributions are merely special cases. Doing so will allow researchers to better approximate the true DGP, make better predictions, and extract more information from their observations. If researchers adopt distributional assumptions for which other distributions are merely special cases, then researcher flexibility is greatly decreased; in such a case, the normal distribution is a special case of the skew-normal distribution, and thus adopting a skew-normal assumption by default may decrease analytic flexibility relative to other methods for managing skewed observations (Gelman \& Loken, 2013).

For this particular paper, we focused on the skew-normal distribution as a way of handling asymmetry of observations 
from two groups. The skew-normal distribution can of course be used in linear regression (skewed residuals), latent variable models (skewed residuals or skewed latent variables causing skewed marginal variables), random effects models (skewed residuals or skewed random effects distributions), and several other models common to psychologists. Moreover, the skew-normal is merely one of several skew-elliptical distributions. Just as the Student $\mathrm{t}$ distribution can be used for robust estimation (e.g., modeling outliers; Kruschke, 2012; Williams \& Martin, in preparation), the Skew-T distribution (Arellano-Valle \& Azzalini, 2013) can be used for robust analysis of asymmetric data.

\section{Implications: Methodological}

The present paper is of methodological importance for several reasons. Bayesian approaches are becoming more common in psychology, especially those using Bayes factors. These methods have been posited as offering advantages compared to classical methods, with some authors explicitly stating that the goals between approaches - frequentist vs. Bayesian - differ. However, goals can take on a variety of definitions and examining frequentist properties of Bayesian models has a long tradition in statistical oriented fields. We view frequentist quantities as extremely useful for characterizing performance of Bayesian models in repeated use and improving them, even if Bayesian inference does not depend on assumed sampling distributions for inference. That is, although Bayesian inference does not depend on inference through sampling error and sampling distributions, understanding how Bayesian-derived estimates and decisions perform in repeated use is important for the propagation and acceptance of Bayesian methods. Some psychologists may be averse to the notion of combining frequentist metrics with Bayesian inferential methods. This paper demonstrates that this rift need not exist, as the frequentist properties of a Bayesian inferential method are orthogonal to the frequentist inferential paradigm. Moreover, for researchers concerned about frequentist properties of Bayesian models, this paper should alleviate some concern.

Second, concerns have been raised about subjectivity in Bayesian methods. A seemingly common argument for frequentism is that Bayesianism is subjective and frequentism is objective. Outside of simulation, the hypothetical sampling distribution is an unobserved, assumed distribution of data, and thus error rates are no more objective or known than a Bayesian method with assumed distributions on parameters (Jaynes, 1976). Instead, we suggest that inferential frameworks be judged by their performance when the truth is known, and when the methods are employed as intended In this case, the Bayesian methods indeed enjoy the frequentist properties that non-Bayesians may be concerned about. Regardless of inferential framework, statistical modeling consists of many choices and assumptions. In the Bayesian framework, the assumptions and priors are made explicit in the model definition, inviting critiques and model evaluation. Moreover, the only requirement for priors is that one express prior information or plausibility as some probability function in a defensible manner. Should a researcher objectively have little to no information, then at minimum one may express that extreme state of uncertainty using a non-informative prior. Indeed, maximum likelihood estimates are mathematically identical to Bayesian MAP estimates under an implicit uniform prior, regardless of whether such a prior is defensible: $p(\theta \mid D)=p(D \mid \theta) \times \frac{C}{A}, \max (L(\theta \mid D))=\max \left(p(D \mid \theta) \times \frac{C}{A}\right)$, where $\mathrm{A}$ and $\mathrm{C}$ are constants.

Third, because it requires no derivation of or assumption about sampling distributions for inference, Bayesian modeling allows for fitting arbitrarily complex and novel theoretical models that are not possible with classical approaches. Although the present paper examined a simple comparison between two-groups, this can easily be extended to more complex situations. For example, there are at least two examples of random effects being modeled with a skewnormal distribution using Bayesian methods (Arellano-Valle, Bolfarine, \& Lachos, 2007; Arellano, Bolfarine, \& Lachos, 2005; Rastegaran \& Zadkarami, 2015). These approaches can be easily extended to allow for heteroskedastic variances, and this is not currently available with frequentist methods (e.g., maximum likelihood). In addition to structurally complex models, Bayesian modeling flexibly permits the use of hundreds of likelihoods that can feasibly generate observed data. For example, experiments with reaction time data have been analyzed using non-Gaussian distributions without transformation procedures (Ratcliff, 1979). Although the skewnormal has not been used for reaction times (to our knowledge), our simulations show that, for heavily skewed data, the skew-normal has optimal performance that can allow for detecting an effect with fewer subjects, and nevertheless will perform adequately with minor or no skew.

\section{Implications: Reproducible research}

Addressing the so-called replication crisis has dominated psychology for several years (Kerr, 1998; Simmons et al., 2011; Klein et al., 2014; Open Science Collaboration, 2015; Gelman \& Loken, 2013; Masicampo \& Lalande, 2012; Francis, 2012). Even if we assume that the reported results in the primary studies are unbiased, using an inappropriate statistical analysis can produce results that are artificially difficult to replicate for two reasons. First, when an analysis is underpowered, any effects that pass a "significance filter" must necessarily be overestimates of the true effect - The more underpowered a study, the greater the estimate bias must necessarily be if the detected effect is "significant" (Loken \& Gelman, 2017). When a replicator wishes to plan a replication study, they may use this overestimate as a guide in an a-priori power analysis. Given that the effect is an overestimate, the 
power analysis will necessarily produce a sample size that is below the power goal of the replicator, and may fail to replicate. Second, when a researcher wishes to attempt a replication, using a mismatching model reduces the power to detect any effect, and thus the ability to replicate effects is diminished. Although considerable effort has gone into improving power in psychology, the focus is generally on increasing sample size, which is not always feasible for some research questions. In contrast, far less attention has been given to statistical methodology. We demonstrated that models which best match the characteristics of the data have the greatest power. If paired with a significance filter, a better powered analytic method will produce less biased estimates than underpowered analytic methods, which benefits replicators' ability to plan a replication and detect the effect a second time.

It has been argued that most research findings are false, the literature has too many significant findings due to publication bias, researchers engage in questionable research practices, and that post-hoc justified data-dependent analytic decisions can make findings difficult to replicate (Francis, 2012; Masicampo \& Lalande, 2012; Kerr, 1998; Simmons et al., 2011; Gelman \& Loken, 2013). Importantly, while our results do not address these issues, they still allow for limiting researcher degrees of freedom. Data altering procedures entail making data-dependent decisions, each of which is unaccounted for in sampling distributions used for classical inference, and thus cause inflated error rates. Bayesian modeling overcomes these issues by allowing the model to calibrate to the observed data. This reduces the need to alter one's data, while also providing optimal error rates and power. Instead of choosing to adjust observations by some random variable and choosing some transformation procedure, both choices which depend on a realized dataset, researchers can instead choose to use less restrictive assumptions, such as using a skew-normal to permit normality or skewness.

Moreover, ensuring that a model can feasibly generate observed data should be established before drawing inferences about the parameters. Should a posterior predictive check suggest that a model cannot feasibly generate the observed data, any inference derived from the estimated parameters of such a model must be questioned. Yet currently, posterior predictive checking through Bayesian models is not common practice in psychology, and effects may be difficult to replicate simply because the effects are estimated with untenable assumptions (e.g., normality, homoskedasticity).

\section{Final Thoughts}

The heterogenous skew-normal model could be improved. One future possibility is to place a hierarchical prior on the groups' respective scale parameters, such that if the groups have homogenous variance, then the model can essentially collapse to a homogenous variance model. Such a model would likely have less power than the homogenous skewnormal model, but more power than the other models when homogenous variance is tenable. In the simple case of two groups with skewed residuals, this random effects heterogenous skew-normal model may provide an even safer default for those who are uncomfortable deciding between the homogenous or heterogenous skew-normal models.

The authors do not wish to suggest that all data transformations are ill-guided. They are not; many data transformations are theoretically motivated and useful. In the case where one wishes to model multiplicative relationships, a log transform can and should probably be used. If one is confident that certain observations are derived from a separate DGP entirely (e.g., a mistaken response, a miscoded value, a programming error), excluding such outliers for the purpose of inferring about the DGP of interest should be encouraged (or DGPs can be simultaneously modeled using mixture modeling). Linearly transforming variables to a more interpretable scale, or centering to allow for more intuitive interpretations of moderators and intercepts, is useful and should be encouraged.

However, transforming data to satisfy atheoretical or computationally convenient assumptions can be avoided, and doing so allows for better prediction, more information about the DGP, greater power, and less bias. In the case of even minutely asymmetric, non-normal data, one may simply exchange the normality assumption with a Skew-Normality assumption. The skew-normal distribution is not reserved for only severely and obviously skewed data, and can be used without loss even when skew is imperceptible, but nevertheless possibly present. Moreover, the skew-normal can nevertheless be used following a meaningful, theoretically motivated transformation - For example, a multiplicative, log-transformed model may have asymmetric residuals, for which the Skew-Normal may be adequate. In sum, if theory dictates distributional assumptions or transformations are theoretically useful, then use them; however, if data transformations are conducted merely to meet an apparently untenable assumption, then we argue instead to replace the assumption in the model with one that is tenable, and Bayesian modeling easily permits such liberties.

Psychologists need not be constrained by normality assumptions. As scientists, our goal is to obtain data and make inferences from the data about the process that produced such data. Should the DGP not produce perfectly symmetric normally distributed values, our models for inferring about the DGP should permit the possibility to improve inference. In the particular case of skew, we recommend exploring the Skew-Normal distribution for your analytic needs. Should the Skew-Normal be insufficient, we recommend exploring the Skew-T distribution, or other distributions that can explicitly model skewed data (Ratcliff, 1979; Arellano-Valle \& Azzalini, 2013). Doing so can improve your inference, pro- 
vide more power, improve prediction, and minimize flexibility in decisions about data manipulations.

\section{References}

Arellano, R., Bolfarine, H., \& Lachos, V. H. (2005). Skewnormal linear mixed models. Fournal of Data Science, 3, 415-438. doi:10.1080/02664760701236905

Arellano-Valle, R. B. \& Azzalini, A. (2013). The centred parameterization and related quantities of the skew-t distribution. Journal of Multivariate Analysis, 113, 73-90. doi:10.1016/j.jmva.2011.05.016

Arellano-Valle, R. B., Bolfarine, H., \& Lachos, V. H. (2007). Bayesian inference for skew-normal linear mixed models. Journal of Applied Statistics, 34(6), 663-682. doi:10. 1080/02664760701236905

Arellano-Valle, R. B., Del Pino, G., \& San Martín, E. (2002). Definition and probabilistic properties of skewdistributions. Statistics and Probability Letters, 58(2), 111-121. doi:10.1016/S0167-7152(02)00088-3

Arellano-Valle, R. B. \& Genton, M. G. (2005). On fundamental skew distributions. Fournal of Multivariate Analysis, 96(1), 93-116. doi:10.1016/j.jmva.2004.10.002

Azzalini, A. (1985). A class of distributions which includes the normal ones. Scandinavian fournal of Statistics, 12(2), 171-178.

Azzalini, A. (2017). The $R$ package sn: The skew-normal and skew-t distributions (version 1.5-0). Università di Padova, Italia. Retrieved from http://azzalini.stat.unipd. it/SN

Azzalini, A. \& Capitanio, A. (1999). Statistical applications of the multivariate skew normal distribution. Fournal of the Royal Statistical Society: Series B (Methodological), 61(3), 579-602. arXiv: 0911.2093v1

Azzalini, A. \& Capitanio, A. (2003). Distributions generated by perturbation of symmetry with emphasis on a multivariate skew $\mathrm{t}$-distribution. Fournal of the Royal Statistical Society: Series B (Methodological), 65(2), 367-389. arXiv: 0911.2342

Azzalini, A. \& Dalla Valle, A. (1996). The multivariate skewnormal distribution. Biometrika, 83(4), 715-726.

Berger, J. O., Boukai, B., Wang, Y., Lindley, D. V., Louis, T. A., Hinkley, D., ... Wang, Y. (1997). Unified frequentist and Bayesian testing of a precise hypothesis. Statistical Science, 12(3), 133-160. doi:10.1214/ss/1030037904

Bhadra, A., Datta, J., Polson, N. G., \& Willard, B. (2015). The horseshoe+ estimator of ultra-sparse signals. arXiv: 1502.00560

Box, G. E. P. (1980). Sampling and Bayes' inference in scientific modelling and robustness. Journal of the Royal Statistical Society: Series A (General), 143(4), 383-430. doi:10.2307/2982063
Box, G. E. P. \& Cox, D. R. (1964). An analysis of transformations. Fournal of the Royal Statistical Society: Series B (Methodological), 26(2), 211-252.

Bürkner, P.-C. (in press). brms: An R package for Bayesian multilevel models using stan. Fournal of Statistical Software.

Carpenter, B., Gelman, A., Hoffman, M. D., Lee, D., Goodrich, B., Betancourt, M., ... Riddell, A. (2017). Stan: A probabilistic programming language. fournal of Statistical Software, 76(1). doi:10.18637/jss.v076.i01

Carvalho, C. M., Polson, N. G., \& Scott, J. G. (2010). The horseshoe estimator for sparse signals. Biometrika, 97(2), 465-480. doi:10.1093/biomet/asq017

Chung, Y., Rabe-Hesketh, S., \& Choi, I.-H. (2013). Avoiding zero between-study variance estimates in randomeffects meta-analysis. Statistics in Medicine, 32(23), 4071-4089. doi:10.1002/sim.5821

Chung, Y., Rabe-hesketh, S., Gelman, A., Dorie, V., \& Liu, J. (2012). Avoiding boundary estimates in linear mixed models through weakly informative priors. U.C. Berkeley Division of Biostatistics Working Paper Series, 125. Retrieved from http://stat.columbia.edu/\%7B \% 7Djcliu/paper/HierarchicalPrior.pdf

Diaconis, P. \& Freedman, D. (1986). On the consistency of Bayes estimates. Annals of Statistics, 14(1), 1-26. doi:10. 1214/aos/1176348654

Feng, C., Wang, H., Lu, N., Chen, T., He, H., Lu, Y., \& Tu, X. M. (2014). Log-transformation and its implications for data analysis. Biostatistics in psychiatry, 26(2). doi:10.3969/j. issn.1002-0829.2014.02.009

Fernandez, C. \& Steel, M. F. J. (1998). On Bayesian modeling of fat tails and skewness. Fournal of the American Statistical Association, 93(441), 359-371. doi:10.1080/ 01621459.1998.10474117

Francis, G. (2012). The psychology of replication and replication in psychology. Perspectives on Psychological Science, 7(6), 585-594. doi:10.1177/1745691612459520

Gelman, A. (2003). A Bayesian formulation of exploratory data analysis and goodness-of-fit testing. International Statistical Review, 71(2), 369-382. doi:10.1111/j.17515823.2003.tb00203.x

Gelman, A. (2006). Prior distribution for variance parameters in hierarchical models. Bayesian Analysis, 1(3), 515533. arXiv: $1011.1669 \mathrm{v} 3$

Gelman, A. \& Loken, E. (2013). The garden of forking paths: Why multiple comparisons can be a problem, even when there is no "fishing expedition" or "p-hacking" and the research hypothesis was posited ahead of time. Retrieved from http : / www . stat . columbia . edu / \%7Egelman/research/unpublished/p_hacking.pdf

Jaynes, E. T. (1976). Confidence intervals vs. Bayesian intervals. In W. L. Harper \& C. A. Hooker (Eds.), Foundations of probability theory, statistical inference, and statistical 
theories of science (Vol. 2, pp. 175-257). doi:10.1007/97894-010-1436-6_6

Kerr, N. L. (1998). Harking: Hypothesizing after the results are known. Personality and Social Psychology Review, 2(3), 196-217. doi:10.1207/s15327957pspr0203_4

Klein, R. A., Ratliff, K. A., Vianello, M., Adams, R. B., Bahník, Š., Bernstein, M. J., ... Nosek, B. A. (2014). Investigating variation in replicability: A "many labs" replication project. Social Psychology, 45(3), 142-152. doi:10.1027/ 1864-9335/a000178

Kruschke, J. K. (2012). Bayesian estimation supersedes the t test. Fournal of Experimental Psychology: General, 142(2), 573-603. doi:10.1037/a0029146

Lin, C., Gelman, A., Price, P. N., \& Krantz, D. H. (1999). Analysis of local decisions using hierarchical modeling, applied to home radon measurement and remediation. Statistical Science, 14(3), 305-328.

Little, R. J. (2006). Calibrated Bayes: A Bayes/frequentist roadmap. The American Statistician, 60(3), 213-223. doi:10.1198/000313006X117837

Loken, E. \& Gelman, A. (2017). Measurement error and the replication crisis. Science, 355(6325), 584-585. doi:10. 1126/science.aal3618

Martin, S. R. \& Masicampo, E. J. (2013). Implicit attitudes toward science and religion. Unpublished Manuscript.

Masicampo, E. J. \& Lalande, D. R. (2012). A peculiar prevalence of $\mathrm{p}$ values just below .05. Quarterly fournal of Experimental Psychology, 65(11), 2271-2279. doi:10.1080/ 17470218.2012 .711335

Muthén, B. \& Asparouhov, T. (2011). Bayesian SEM : A more flexible representation of substantive theory. Psychological Methods, 17(3), 313-335. doi:10.1037/a0026802

Nicolaou, A. (1993). Bayesian intervals with good frequentist behaviour in the presence of nuisance parameters. Journal of the Royal Statistical Society: Series B (Methodological), 55(2), 377-390. doi:10.2307/2346199

Open Science Collaboration. (2015). Estimating the reproducibility of psychological science. Science, 349(6251). doi:10.1126/science.aac4716

Park, T. \& Casella, G. (2008). The Bayesian lasso. Journal of the American Statistical Association, 103(482), 681-686. doi:10.1198/016214508000000337

Peers, H. (1968). Confidence properties of Bayesian interval estimates. Fournal of the Royal Statistical Society: Series $B$ (Methodological), 30(3), 535-544.

Rastegaran, A. \& Zadkarami, M. R. (2015). A skew-normal random effects model for longitudinal ordinal categorical responses with missing data. Journal of Applied Statistics, 42(1), 114-126. doi:10.1080/02664763.2014. 938223

Ratcliff, R. (1979). Group reaction time distributions and an analysis of distribution statistics. Psychological Bulletin, $86(3), 446$.
Severini, T. A. (1993). Bayesian interval estimates which are also confidence intervals. Fournal of the Royal Statistical Society: Series B (Methodological), 55, 533-540.

Simmons, J. P., Nelson, L. D., \& Simonsohn, U. (2011). Falsepositive psychology: Undisclosed flexibility in data collection and analysis allows presenting anything as significant. Psychological Science, 22(11), 11359-11366. doi:10.1177/0959797611417632

Stan Development Team. (2016). RStan: The R interface to Stan. R package version 2.14.1. Retrieved from http: //mc-stan.org/

Thatcher, A. R. (1964). Relationships between Bayesian and confidence limits for predictions. Fournal of the Royal Statistical Society: Series B (Methodological), 26(2), 176210. doi: $10.2307 / 2984417$

Vehtari, A., Gelman, A., \& Gabry, J. (2015). Practical Bayesian model evaluation using leave-one-out cross-validation and waic. arXiv: 1507.04544

Venables, W. N. \& Ripley, B. D. (2002). Modern applied statistics with $S$ (Fourth). ISBN 0-387-95457-0. New York: Springer. Retrieved from http://www.stats.ox.ac.uk/ pub/MASS4

Williams, D. R. \& Martin, S. R. (in preparation). Rethinking robust statistics with modern bayesian methods.

Woodroofe, M. (1976). Frequentist properties of Bayesian sequential tests. Biometrika, 63(1), 101-110. doi:10.1093/ biomet/63.1.101 
Appendix A

Example R Code

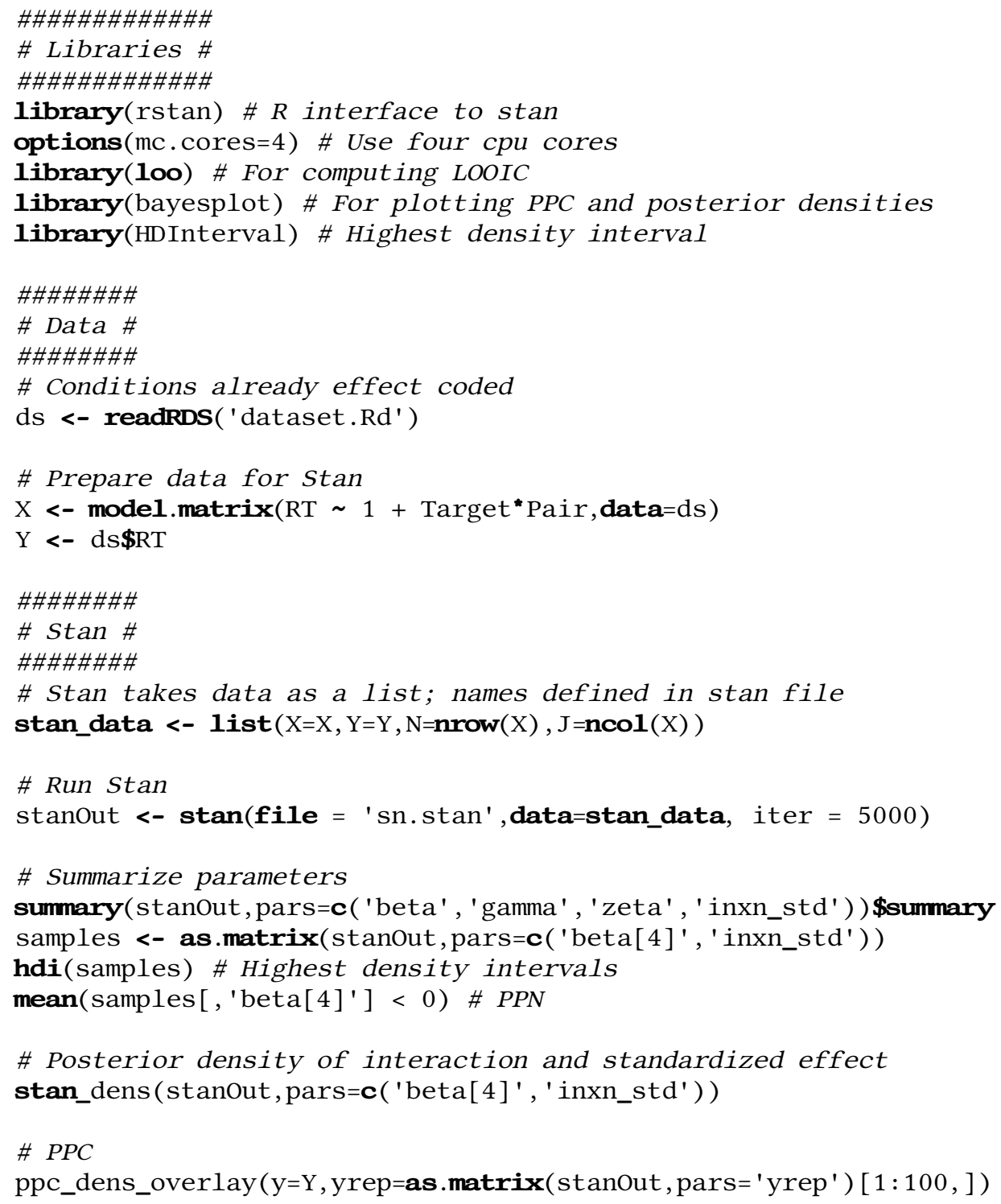



)

prior(normal $(1000,2000)$, class = 'Intercept') \# The intercept for RT (marginal mean)

brmOut <- brm(brmModel, data=ds, prior=brmPriors, iter = 5000)

\# Summarize parameters

brmOut

hdi(as.matrix(brmOut\$fit, pars= 'b_Target 1 : Pair1' ) )

\# Posterior density of interaction

plot(brmout, pars= 'b_Target 1 : Pair1')

\# PPC

pp_check(brmOut, nsamples=100)

\# LOO

100 (brmOut)

\#\#\#\#\#\#

\# SN \#

\#\#\#\#\#\#

\# NOTE: This does not enable heterogenous skew or variance

\# Only use this if homogeneity of skew and variance is assumed and tenable

library (sn)

snout <- selm(RT Target*Pair, data=ds)

summary (snOut) 
Appendix B

Example Stan Model Code (sn.stan)

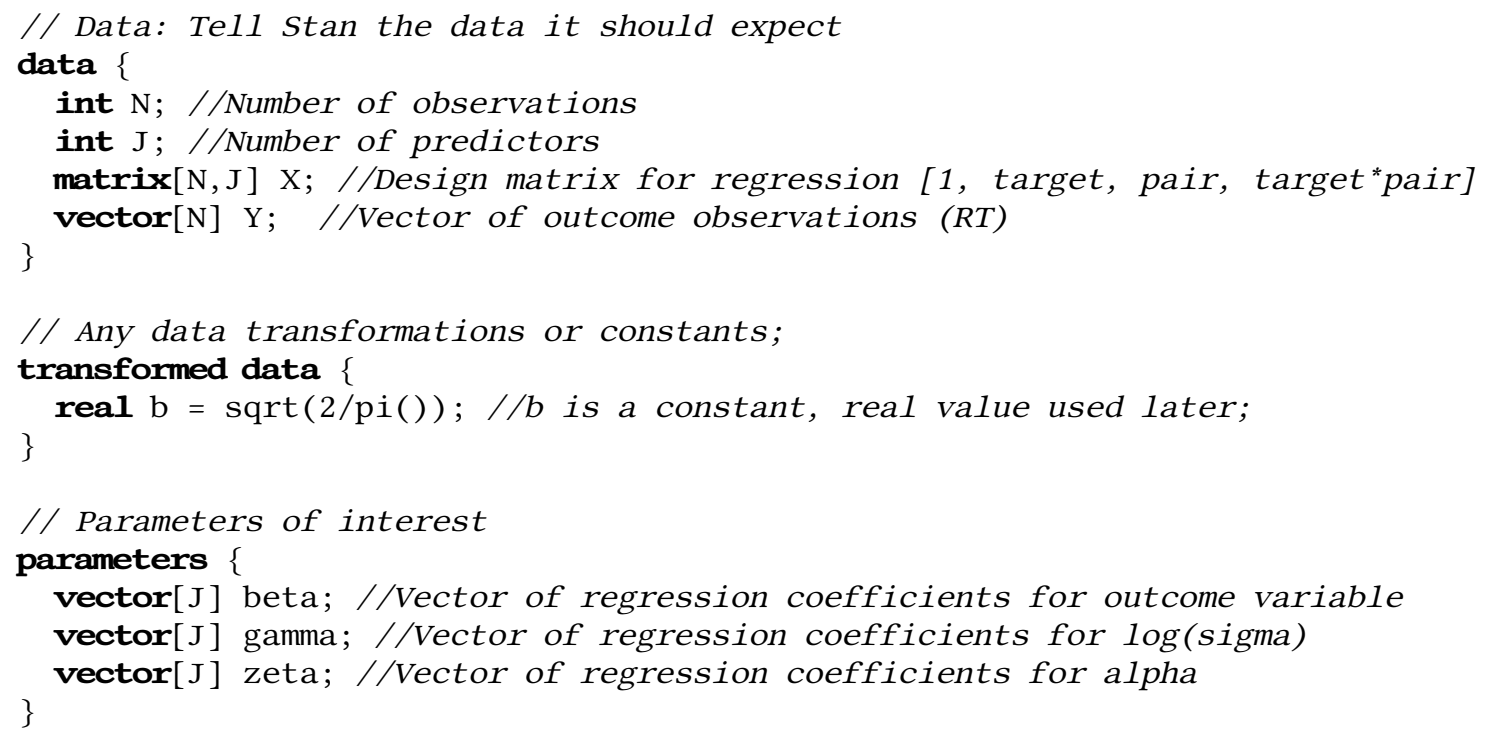


zeta normal $(0,8)$; //Identifying prior to constrain to discernible values of alpha beta[1] normal(1000,2000); //Global mean prior set to 1000ms with sd of 2000 beta $[2: \mathrm{J}] \sim \operatorname{normal}(0,1000) ; / /$ Wide, less informative prior for group differences

// Likelihood

$\mathrm{Y}$ skew_normal(xi, omega,alpha);

\}

// Define any quantity of interest as a function of model information generated quantities \{

vector[N] yrep; // Posterior predictive density; for PPC

vector[N] log_lik; // Log Likelihood for each observation; for LOOIC

real inxn_std; // Standardized effect of interaction for d-statistic

// With effect coding, equivalent to d_1(1) - d_1(-1)

inxn_std $=4{ }^{*} \operatorname{beta}[4] / \exp ($ gamma[1] $)$;

\{ //Unsaved variables, used for computing yrep and log_lik, same as model block vector $[\mathrm{N}]$ muHat $=\mathrm{X}^{*}$ beta;

vector $[\mathrm{N}] \operatorname{logSigma}=\mathrm{X}^{*}$ gamma;

vector $[\mathrm{N}]$ alpha $=\mathrm{X}^{*}$ zeta;

vector $[\mathrm{N}]$ sigma;

vector $[\mathrm{N}]$ delta;

vector $[\mathrm{N}]$ omega;

vector $[\mathrm{N}] \mathrm{xi}$;

for $(\mathrm{n}$ in $1: \mathrm{N})\{$

$\operatorname{sigma}[\mathrm{n}]=\exp (\operatorname{logSigma}[\mathrm{n}]) ;$

$\operatorname{delta}[\mathrm{n}]=\operatorname{alpha}[\mathrm{n}] / \operatorname{sqrt}\left(1+\operatorname{alpha}[\mathrm{n}]^{\wedge} 2\right) ;$

$\operatorname{omega}[n]=\operatorname{sigma}[n] / \operatorname{sqrt}\left(1-\operatorname{delta}[n]^{\wedge} 2^{*} b^{\wedge} 2\right) ;$

$\mathrm{xi}[\mathrm{n}]=\operatorname{muHat}[\mathrm{n}]-\operatorname{omega}[\mathrm{n}]^{*} \operatorname{delta}[\mathrm{n}]{ }^{*} \mathrm{~b}$;

// Likelihood of $Y[n]$ given predicted $x i$, omega, and alpha

$\log \_1 \mathrm{ik}[\mathrm{n}]=$ skew_normal_lpdf $(\mathrm{Y}[\mathrm{n}] \mid \mathrm{xi}[\mathrm{n}]$, omega[n], alpha[n]);

// Given predicted xi, omega, alpha, generate replicate observed value

$\operatorname{yrep}[\mathrm{n}]=$ skew_normal_rng $(\mathrm{xi}[\mathrm{n}]$, omega $[\mathrm{n}], \mathrm{alpha}[\mathrm{n}])$;

\} 